Open J. Math. Sci., Vol. 1(2017), No. 1, pp. 62-71

Website: https://pisrt.org/psr-press/journals/oms/

ISSN: 2523-0212 (Online) 2616-4906 (Print)

http://dx.doi.org/10.30538/oms2017.0007

\title{
COMPUTING TOPOLOGICAL INDICES OF HEX BOARD AND ITS LINE GRAPH
}

\author{
HAFIZ MUTEE UR REHMAN ${ }^{1}$, RIFFAT SARDAR, ALI RAZA
}

\begin{abstract}
A topological index is a real number related to a molecular graph, which is a graph invariant. Uptill now there are several topological indices are defined. Some of them are distance based while the others are degree based, all have found numerous applications in pharmacy, theoretical chemistry and especially in QSPR/QSAR research. In this paper, we compute some degree based topological indices i.e some versions of Zagreb indices, Randić index, General sum connectivity index and GA index of Hex board and of its line graph.
\end{abstract}

AMS Mathematics Subject Classification: 05C12, 05C90.

Key words and phrases: degree; topological index; Zagreb index.

\section{Introduction and Preliminaries}

Mathematical chemistry is the branch of theoretical chemistry in which we discuss and predict the behavior of mathematical structure by using mathematical tools. There is lot of research which is done in this area in the last few decades. This theory contributes a major role in the field of chemical sciences.

Let $G$ be the molecular graph in which $V(G)$ represents the set of vertices corresponds the atoms and $E(G)$ the set of edges to the chemical bonds. A line graph $L(G)$ of a simple graph $G$ is obtained by associating a vertex with each edge of the graph and connecting two vertices with an edge if and only if the corresponding edges of $G$ have a vertex in common.

The very first topological index Randić index introduced by Milan Randić in

Received 13 August 2017. Revised 29 September 2017.

1 Corresponding Author

(C) 2017 Hafiz Mutee ur Rehman, Riffat Sardar, Ali Raza. This is an open access article distributed under the Creative Commons Attribution License, which permits unrestricted use, distribution, and reproduction in any medium, provided the original work is properly cited. 
1975 (see [1]). and is defined as:

$$
R(G)=\sum_{u v \in E(G)} \frac{1}{\sqrt{d_{u} d_{v}}} .
$$

Later, this index was generalized by Bollobás \& Erdös( see [2]) to the following form for any real number $\alpha$, and named the general Randić index:

$$
R_{\alpha}(G)=\sum_{u v \in E(G)}\left[d_{u} d_{v}\right]^{\alpha} .
$$

The Zagreb indices were first introduced by Gutman in [3], they are important molecular descriptors and have been closely correlated with many chemical properties (see [4]) and defined as:

$$
\begin{aligned}
& M_{1}(G)=\sum_{u \in V(G)} d_{u}^{2} \quad \text { and } \\
& M_{2}(G)=\sum_{u v \in E(G)} d_{u} d_{v} .
\end{aligned}
$$

The third Zagreb index, introduced by Fath-Tabar in [5]. This index is defined as follows:

$$
M_{3}(G)=\sum_{u v \in E(G)}\left|d_{u}-d_{v}\right| .
$$

The hyper-Zagreb index was first introduced in [6]. This index is defined as follows:

$$
H M(G)=\sum_{u v \in E(G)}\left(d_{u}+d_{v}\right)^{2} .
$$

The Atom-Bond Connectivity index (ABC), introduced by Estrada, et al. in [7] and applied up until now to study the stability of alkanes and the strain energy of cycloalkanes. The ABC index of $G$ is defined as:

$$
A B C(G)=\sum_{u v \in E(G)} \sqrt{\frac{d_{u}+d_{v}-2}{d_{u} d_{v}}} .
$$

For more details see the article [8]. In 2010, the general sum-connectivity index $\chi(G)$ has been introduced in [9]. For more detail on sum connectivity we refer the articles $[9,10]$. This index is defined as follows:

$$
\chi(G)=\sum_{u v \in E(G)} \frac{1}{\sqrt{d_{u}+d_{v}}} .
$$

Vukićević and Furtula introduced the geometric arithmetic (GA) index in [11]. The GA index for $G$ is defined by

$$
G A(G)=\sum_{u v \in E(G)} \frac{2 \sqrt{d_{u} d_{v}}}{d_{u}+d_{v}} .
$$


Inspired by the work on the $\mathrm{ABC}$ index, Furtula et al. proposed the following modified version of the $\mathrm{ABC}$ index and called it as augmented Zagreb index (AZI) in [12]. This index is defined as follows:

$$
A Z I(G)=\sum_{u v \in E(G)}\left(\frac{d_{u} d_{v}}{d_{u}+d_{v}-2}\right)^{3} .
$$

The hexagonal and honeycomb networks have also been recognized as crucial evolutionary biology, in particular for the evolution of cooperation, where the overlapping triangles are vital for the propagation of cooperation in social dilemmas. Relevant research that applies this theory and which could benefit further from the insights of the new research in (see [13]).

The following lemma is helpful for computing the degree of a vertex of line graph.

Lemma 1.1. Let $G$ be a graph with $u, v \in V(G)$ and $e=u v \in E(G)$. Then:

$$
d_{e}=d_{u}+d_{v}-2 .
$$

Lemma 1.2. [14] Let $G$ be a graph of order $p$ and size $q$, then the line graph $L(G)$ of $G$ is a graph of order $p$ and size $\frac{1}{2} M_{1}(G)-q$.

\section{Topological indices of Hex board}

In this section we will compute the topological indices of Hex board.

Theorem 2.1. Let $G$ be the Hex board $H_{n}$. Then

(1) $M_{1}(G)=36 n^{2}-80 n+42$;

(2) $M_{2}(G)=108 n^{2}-320 n+232$;

(3) $M_{3}(G)=-16 n+22$;

(4) $H M(G)=432 n^{2}-1248 n+886$;

(5) $R(G)=\frac{1}{2} n^{2}+\frac{1}{12}(8 n-20) \sqrt{6}-\frac{5}{3} n+\frac{4}{3} \sqrt{2}+\frac{2}{3} \sqrt{3}+1$;

(6) $A B C(G)=\frac{1}{6}\left(3 n^{2}-16 n+21\right) \sqrt{10}+\frac{1}{3}(8 n-20) \sqrt{3}+\frac{1}{4}(4 n-10) \sqrt{6}+$ $\frac{1}{3} \sqrt{14}+\frac{2}{3} \sqrt{15}+2 \sqrt{2}$

(7) $\chi(G)=\frac{1}{6}\left(3 n^{2}-16 n+21\right) \sqrt{3}+\frac{1}{10}(8 n-20) \sqrt{10}+\frac{1}{4}(4 n-10) \sqrt{2}+\frac{2}{3} \sqrt{6}+$ $\frac{4}{7} \sqrt{7}+\frac{2}{3}$

(8) $G A(G)=3 n^{2}+\frac{2}{5}(8 n-20) \sqrt{6}-12 n+4 \sqrt{2}+\frac{16}{7} \sqrt{3}+11$;

(9) $A Z I(G)=\frac{17496}{125} n^{2}-\frac{1534424}{3375} n+\frac{429997724}{1157625}$.

Proof. The graph $G$ for $n=4$ is shown in Figure 1. It is easy to see that the order of $G$ is $n^{2}$ out of which 2 vertices are of degree 2, 2 vertices are of degree 3, $4(n-2)$ vertices are of degree 4 and $n^{2}-4(n-1)$ vertices are of degree 6 and $G$ has size $3 n^{2}-4 n+1$. We partition the size of $G$ into edges of the type $E_{\left(d_{u}, d_{v}\right)}$ where $u v$ is an edge. In $G$, we get edges of the type $E_{(2,4)}, E_{(3,4)}, E_{(3,6)}, E_{(4,4)}$, $E_{(4,6)}$ and $E_{(6,6)}$. The number of edges of these types are given in the Table 1. Then we obtain the required results by using Table1 as follows: 


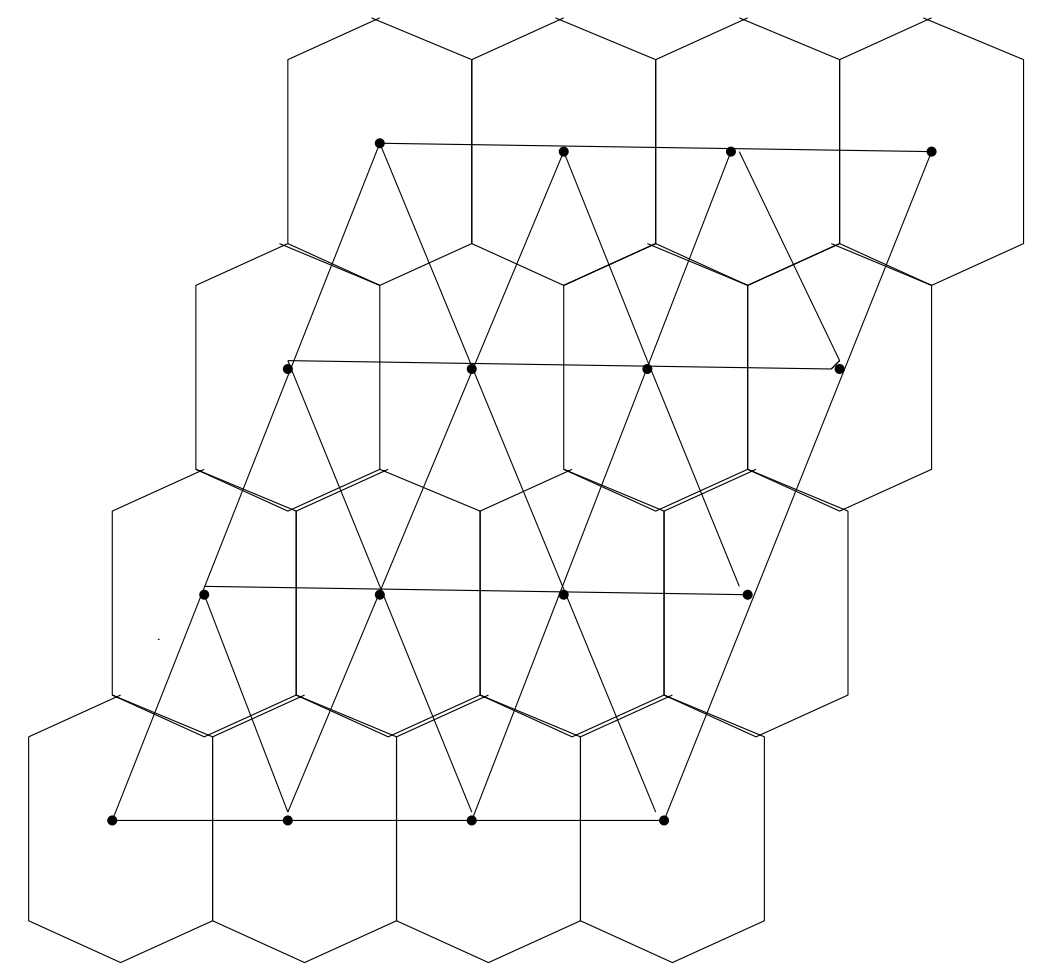

Figure 1. Hex board with center dots.

(1) $M_{1}(G)=\sum_{u v \in E(G)}\left[d_{u}+d_{v}\right]$ $=4(2+4)+4(3+4)+2(3+6)+(4 n-10)(4+4)+(8 n-20)(4+6)+$ $\left(3 n^{2}-16 n+21\right)(6+6)$

$=36 n^{2}-80 n+42$.

(2) $M_{2}(G)=\sum_{u v \in E(G)} d_{u} d_{v}$ $=4(2 \times 4)+4(3 \times 4)+2(3 \times 6)+(4 n-10)(4 \times 4)+(8 n-20)(4 \times 6)+$ $\left(3 n^{2}-16 n+21\right)(6 \times 6)$

$=108 n^{2}-320 n+232$.

(3) $M_{3}(G)=\sum_{u v \in E(G)}\left|d_{u}-d_{v}\right|$

$=4(2-4)+4(3-4)+2(3-6)+(4 n-10)(4-4)+(8 n-20)(4-6)+$ $\left(3 n^{2}-16 n+21\right)(6-6)$.

$=-16 n+22$.

(4) $H M(G)=\sum_{u v \in E(G)}\left(d_{u}+d_{v}\right)^{2}$

$=4(2+4)^{2}+4(3+4)^{2}+2(3+6)^{2}+(4 n-10)(4+4)^{2}+(8 n-20)(4+$ $6)^{2}+\left(3 n^{2}-16 n+21\right)(6+6)^{2}$. $=432 n^{2}-1248 n+886$. 
(5) $R(G)=\sum_{u v \in E(G)} \frac{1}{\sqrt{d_{u} d_{v}}}$

$=4\left(\frac{1}{\sqrt{2 \times 4}}\right)+4\left(\frac{1}{\sqrt{3 \times 4}}\right)+2\left(\frac{1}{\sqrt{3 \times 6}}\right)+(4 n-10)\left(\frac{1}{\sqrt{4 \times 4}}\right)+(8 n-20)\left(\frac{1}{\sqrt{4 \times 6}}\right)+$ $\left(3 n^{2}-16 n+21\right)\left(\frac{1}{\sqrt{6 \times 6}}\right)$.

$\frac{1}{2} n^{2}+\frac{1}{12}(8 n-20) \sqrt{6}-\frac{5}{3} n+\frac{4}{3} \sqrt{2}+\frac{2}{3} \sqrt{3}+1$.

(6) $A B C(G)=\sum_{u v \in E(G)} \sqrt{\frac{d_{u}+d_{v}-2}{d_{u} d_{v}}}$

$=4 \sqrt{\frac{2+4-2}{2 \times 4}}+4 \sqrt{\frac{3+4-2}{3 \times 4}}+2 \sqrt{\frac{3+6-2}{3 \times 6}}+(4 n-10) \sqrt{\frac{4+4-2}{4 \times 4}}+(8 n-20) \sqrt{\frac{4+6-2}{4 \times 6}}+$

$\left(3 n^{2}-16 n+21\right) \sqrt{\frac{6+6-2}{6 \times 6}}$.

$=\frac{1}{6}\left(3 n^{2}-16 n+21\right) \sqrt{10}+\frac{1}{3}(8 n-20) \sqrt{3}+\frac{1}{4}(4 n-10) \sqrt{6}+\frac{1}{3} \sqrt{14}+$

$$
\frac{2}{3} \sqrt{15}+2 \sqrt{2}
$$

(7) $\chi(G)=\sum_{u v \in E(G)} \frac{1}{\sqrt{d_{u}+d_{v}}}$

$=4\left(\frac{1}{\sqrt{2+4}}\right)+4\left(\frac{1}{\sqrt{3+4}}\right)+2\left(\frac{1}{\sqrt{3+6}}\right)+(4 n-10)\left(\frac{1}{\sqrt{4+4}}\right)+(8 n-20)\left(\frac{1}{\sqrt{4+6}}\right)+$

$\left(3 n^{2}-16 n+21\right)\left(\frac{1}{\sqrt{6+6}}\right)$.

$=\frac{1}{6}\left(3 n^{2}-16 n+21\right) \sqrt{3}+\frac{1}{10}(8 n-20) \sqrt{10}+\frac{1}{4}(4 n-10) \sqrt{2}+\frac{2}{3} \sqrt{6}+\frac{4}{7} \sqrt{7}+\frac{2}{3}$.

(8) $G A(G)=\sum_{u v \in E(G)} \frac{2 \sqrt{d_{u} d_{v}}}{d_{u}+d_{v}}$

$=4\left(\frac{2 \sqrt{2 \times 4}}{2+4}\right)+4\left(\frac{2 \sqrt{3 \times 4}}{3+4}\right)+2\left(\frac{2 \sqrt{3 \times 6}}{3+6}\right)+(4 n-10)\left(\frac{2 \sqrt{4 \times 4}}{4+4}\right)+(8 n-20)\left(\frac{2 \sqrt{4 \times 6}}{4+6}\right)+$

$\left(3 n^{2}-16 n+21\right)\left(\frac{2 \sqrt{6 \times 6}}{6+6}\right)$.

$=3 n^{2}+\frac{2}{5}(8 n-20) \sqrt{6}-12 n+4 \sqrt{2}+\frac{16}{7} \sqrt{3}+11$.

(9) $A Z I(G)=\sum_{u v \in E(G)}\left(\frac{d_{u} d_{v}}{d_{u}+d_{v}-2}\right)^{3}$

$=4\left(\frac{2 \times 4}{2+4-2}\right)^{3}+4\left(\frac{3 \times 4}{3+4-2}\right)^{3}+2\left(\frac{3 \times 6}{3+6-2}\right)^{3}+(4 n-10)\left(\frac{4 \times 4}{4+4-2}\right)^{3}+(8 n-$ 20) $\left(\frac{4 \times 6}{4+6-2}\right)^{3}+\left(3 n^{2}-16 n+21\right)\left(\frac{6 \times 6}{6+6-2}\right)^{3}$.

$=\frac{17496}{125} n^{2}-\frac{1534424}{3375} n+\frac{429997724}{1157625}$.

TABle 1. The size partition of $G$

\begin{tabular}{|c|c|c|c|}
\hline$\left(d_{u}, d_{v}\right)$ where $u v \in E(G)$ & $(2,4)$ & $(3,4)$ & $(3,6)$ \\
\hline Number of edges & 4 & 4 & 2 \\
\hline$\left(d_{u}, d_{v}\right)$ where $u v \in E(G)$ & $(4,4)$ & $(4,6)$ & $(6,6)$ \\
\hline Number of edges & $4 n-10$ & $8 n-20$ & $3 n^{2}-16 n+21$ \\
\hline
\end{tabular}

\section{Topological indices of line graph of Hex board}

In this section we will compute the topological indices of the line graph of Hex board.

Theorem 3.1. Let $G$ be the line graph of the Hex board $H_{n}$. Then

(1) $M_{1}(G)=300 n^{2}-944 n+722$;

(2) $M_{2}(G)=1500 n^{2}-5616 n+5194$; 


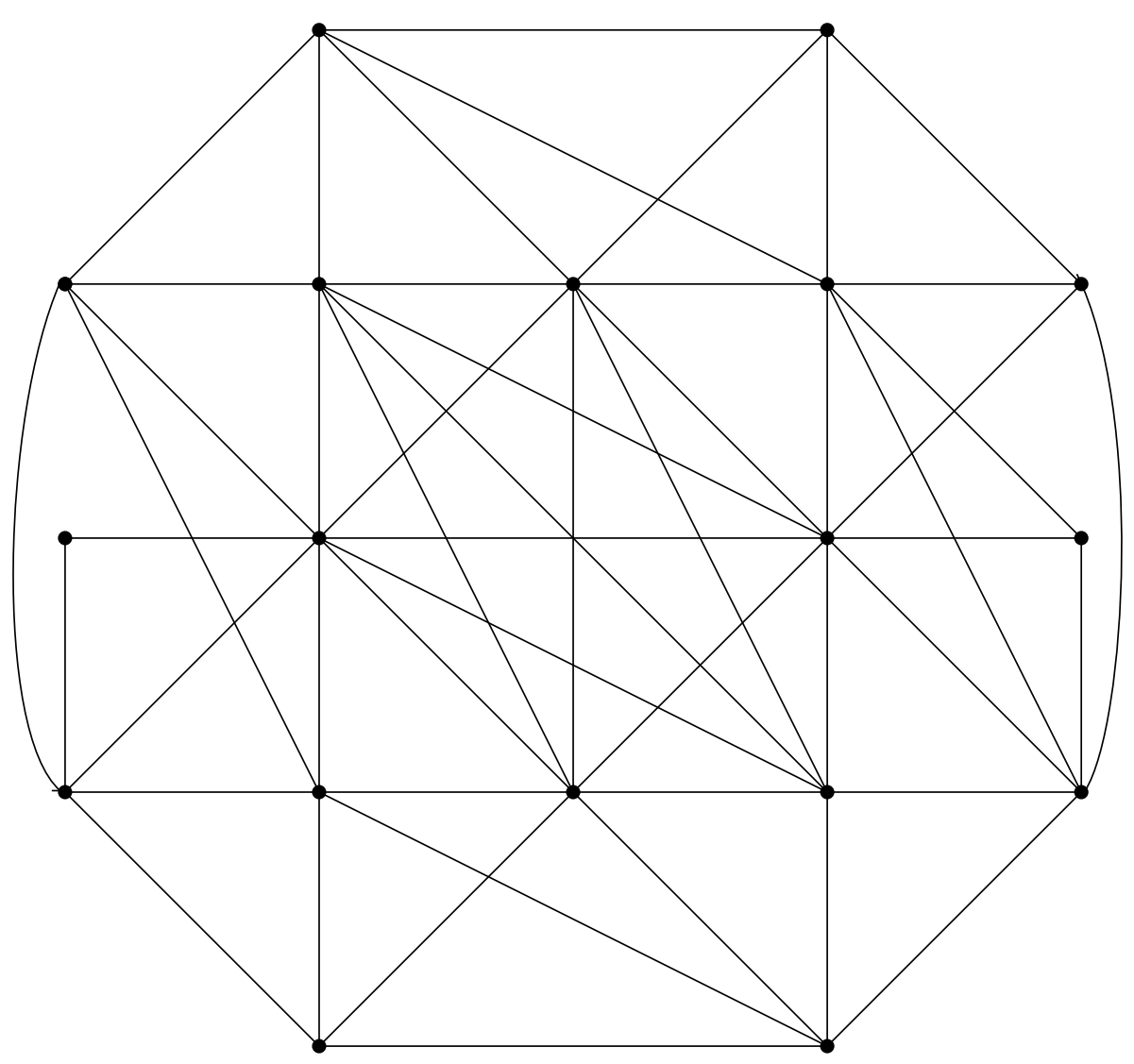

Figure 2. Line graph of hex board with center dots.

(3) $M_{3}(G)=-96 n+206$;

(4) $H M(G)=6000 n^{2}-22272 n+20430$;

(5) $R(G)=\frac{3}{2} n^{2}-\frac{119}{15} n+\frac{1}{20}(32 n-100) \sqrt{5}+\frac{3}{35} \sqrt{100}+\frac{1}{7} \sqrt{14}+\frac{1}{12}(16 n-$ 48) $\sqrt{3}+\frac{2}{5} \sqrt{10}+\frac{4}{35} \sqrt{35}+\frac{2}{15} \sqrt{30}+\frac{1}{2} \sqrt{2}+\frac{2}{3} \sqrt{6}+\frac{247}{20}$;

(6) $A B C(G)=\frac{3}{10}\left(15 n^{2}-96 n+152\right) \sqrt{2}+\frac{1}{5}(32 n-100) \sqrt{5}+\frac{1}{6}(4 n-12) \sqrt{10}+$ $8 n+\frac{1}{8}(8 n-14) \sqrt{14}+\frac{3}{7} \sqrt{42}+\frac{1}{7} \sqrt{82}+\frac{2}{5} \sqrt{110}+\frac{4}{7} \sqrt{14}+\frac{2}{5} \sqrt{30}+\frac{4}{5} \sqrt{2}+$ $\sqrt{5}+\frac{8}{3} \sqrt{3}+\frac{1}{2} \sqrt{6}-24$

(7) $\chi(G)=\frac{1}{10}\left(15 n^{2}-96 n+152\right) \sqrt{5}+\frac{1}{6}(32 n-100) \sqrt{2}+\frac{1}{14}(16 n-48) \sqrt{14}+$ $\frac{1}{6}(4 n-12) \sqrt{3}+2 n-\frac{7}{2}+\frac{6}{17} \sqrt{17}+\frac{4}{15} \sqrt{15}+\frac{8}{13} \sqrt{13}+\frac{4}{11} \sqrt{11}+\frac{4}{3} \sqrt{3}+$ $\sqrt{10}+\frac{1}{2} \sqrt{2}$

(8) $G A(G)=15 n^{2}+\frac{4}{9}(32 n-100) \sqrt{5}+\frac{4}{7}(16 n-48) \sqrt{3}-84 n+\frac{12}{17} \sqrt{70}+$ $\frac{16}{15} \sqrt{14}+\frac{32}{13} \sqrt{10}+\frac{2}{3} \sqrt{35}+\frac{8}{11} \sqrt{30}+\frac{8}{3} \sqrt{2}+\frac{16}{5} \sqrt{6}+130$

(9) $A Z I(G)=\frac{625000}{243} n^{2}-\frac{109249838528}{10418625} n+\frac{1427097955303080667}{133703165756160}$ 
Proof. The graph $G$ for $n=4$ is shown in Figure 2. By using Lemma 1.1, It is easy to see that the order of $G$ is $3 n^{2}-4 n+1$ out of which 4 vertices are of degree 4,4 vertices are of degree 5,2 vertices are of degree 7, $4 n-10$ vertices are of degree $6,8 n-20$ vertices are of degree 8 and $3 n^{2}-16 n+21$ vertices are of degree 10. Therefore by using Lemma 1.2, $G$ has size $15 n^{2}-36 n+20$. We partition the size of $G$ into edges of the type $E_{\left(d_{u}, d_{v}\right)}$ where $u v$ is an edge. In $G$, we get edges of the type $E_{(4,4)}, E_{(4,6)}, E_{(4,8)}, E_{(5,5)}, E_{(5,6)}, E_{(5,7)}, E_{(5,8)}, E_{(6,6)}$, $E_{(6,8)}, E_{(7,8)}, E_{(7,10)}, E_{(8,8)}, E_{(8,10)}$ and $E_{(10,10)}$. The number of edges of these types are given in the Table 2 . Then we obtain the required results by using Table2 as follows:

(1) $M_{1}(G)=\sum_{u v \in E(G)}\left[d_{u}+d_{v}\right]$

$=2(4+4)+8(4+6)+4(4+8)+2(5+5)+4(5+6)+4(5+7)+8(5+$

$8)+(4 n-12)(6+6)+(16 n-48)(6+8)+4(7+8)+6(7+10)+(8 n-$ $14)(8+8)+(32 n-100)(8+10)+\left(15 n^{2}-96 n+152\right)(10+10)$.

$=300 n^{2}-944 n+722$.

(2) $M_{2}(G)=\sum_{u v \in E(G)} d_{u} d_{v}$

$=2(4 \times 4)+8(4 \times 6)+4(4 \times 8)+2(5 \times 5)+4(5 \times 6)+4(5 \times 7)+8(5 \times$

$8)+(4 n-12)(6 \times 6)+(16 n-48)(6 \times 8)+4(7 \times 8)+6(7 \times 10)+(8 n-$ $14)(8 \times 8)+(32 n-100)(8 \times 10)+\left(15 n^{2}-96 n+152\right)(10 \times 10)$.

$=1500 n^{2}-5616 n+5194$.

(3) $M_{3}(G)=\sum_{u v \in E(G)} \mid d_{u}-d_{v}$

$=2(4-4)+8(4-6)+4(4-8)+2(5-5)+4(5-6)+4(5-7)+8(5-$

$8)+(4 n-12)(6-6)+(16 n-48)(6-8)+4(7-8)+6(7-10)+(8 n-$ $14)(8-8)+(32 n-100)(8-10)+\left(15 n^{2}-96 n+152\right)(10-10)$. $=-96 n+206$.

(4) $H M(G)=\sum_{u v \in E(G)}\left(d_{u}+d_{v}\right)^{2}$

$=2(4+4)^{2}+8(4+6)^{2}+4(4+8)^{2}+2(5+5)^{2}+4(5+6)^{2}+4(5+7)^{2}+$ $8(5+8)^{2}+(4 n-12)(6+6)^{2}+(16 n-48)(6+8)^{2}+4(7+8)^{2}+6(7+10)^{2}+$ $(8 n-14)(8+8)^{2}+(32 n-100)(8+10)^{2}+\left(15 n^{2}-96 n+152\right)(10+10)^{2}$. $=6000 n^{2}-22272 n+20430$.

(5) $R(G)=\sum_{u v \in E(G)} \frac{1}{\sqrt{d_{u} d_{v}}}$

$=2\left(\frac{1}{\sqrt{4 \times 4}}\right)+8\left(\frac{1}{\sqrt{4 \times 6}}\right)+4\left(\frac{1}{\sqrt{4 \times 8}}\right)+2\left(\frac{1}{\sqrt{5 \times 5}}\right)+4\left(\frac{1}{\sqrt{5 \times 6}}\right)+4\left(\frac{1}{\sqrt{5 \times 7}}\right)+$ $8\left(\frac{1}{\sqrt{5 \times 8}}\right)+(4 n-12)\left(\frac{1}{\sqrt{6 \times 6}}\right)+(16 n-48)\left(\frac{1}{\sqrt{6 \times 8}}\right)+4\left(\frac{1}{\sqrt{7 \times 8}}\right)+6\left(\frac{1}{\sqrt{7 \times 10}}\right)+$ $(8 n-14)\left(\frac{1}{\sqrt{8 \times 8}}\right)+(32 n-100)\left(\frac{1}{\sqrt{8 \times 10}}\right)+\left(15 n^{2}-96 n+152\right)\left(\frac{1}{\sqrt{10 \times 10}}\right)$. $=\frac{3}{2} n^{2}-\frac{119}{15} n+\frac{1}{20}(32 n-100) \sqrt{5}+\frac{3}{35} \sqrt{100}+\frac{1}{7} \sqrt{14}+\frac{1}{12}(16 n-48) \sqrt{3}+$ $\frac{2}{5} \sqrt{10}+\frac{4}{35} \sqrt{35}+\frac{2}{15} \sqrt{30}+\frac{1}{2} \sqrt{2}+\frac{2}{3} \sqrt{6}+\frac{247}{20}$.

(6) $A B C(G)=\sum_{u v \in E(G)} \sqrt{\frac{d_{u}+d_{v}-2}{d_{u} d_{v}}}$

$=2\left(\sqrt{\frac{4+4-2}{4 \times 4}}\right)+8\left(\sqrt{\frac{4+6-2}{4 \times 6}}\right)+4\left(\sqrt{\frac{4+8-2}{4 \times 8}}\right)+2\left(\sqrt{\frac{5+5-2}{5 \times 5}}\right)+4\left(\sqrt{\frac{5+6-2}{5 \times 6}}\right)+$ $4\left(\sqrt{\frac{5+7-2}{5 \times 7}}\right)+8\left(\sqrt{\frac{5+8-2}{5 \times 8}}\right)+(4 n-12)\left(\sqrt{\frac{6+6-2}{6 \times 6}}\right)+(16 n-48)\left(\sqrt{\frac{6+8-2}{6 \times 8}}\right)+$ 


$$
\begin{aligned}
& 4\left(\sqrt{\frac{7+8-2}{7 \times 8}}\right)+6\left(\sqrt{\frac{7+10-2}{7 \times 10}}\right)+(8 n-14)\left(\sqrt{\frac{8+18-2}{8 \times 8}}\right)+(32 n-100)\left(\sqrt{\frac{8+10-2}{8 \times 10}}\right)+ \\
& \left(15 n^{2}-96 n+152\right)\left(\sqrt{\frac{10+10-2}{10 \times 10}}\right) . \\
& =\frac{3}{10}\left(15 n^{2}-96 n+152\right) \sqrt{2}+\frac{1}{5}(32 n-100) \sqrt{5}+\frac{1}{6}(4 n-12) \sqrt{10}+8 n+ \\
& \frac{1}{8}(8 n-14) \sqrt{14}+\frac{3}{7} \sqrt{42}+\frac{1}{7} \sqrt{82}+\frac{2}{5} \sqrt{110}+\frac{4}{7} \sqrt{14}+\frac{2}{5} \sqrt{30}+\frac{4}{5} \sqrt{2}+\sqrt{5}+ \\
& \frac{8}{3} \sqrt{3}+\frac{1}{2} \sqrt{6}-24
\end{aligned}
$$

(7) $\chi(G)=\sum_{u v \in E(G)} \frac{1}{\sqrt{d_{u}+d_{v}}}$

$=2\left(\frac{1}{\sqrt{4+4}}\right)+8\left(\frac{1}{\sqrt{4+6}}\right)+4\left(\frac{1}{\sqrt{4+8}}\right)+2\left(\frac{1}{\sqrt{5+5}}\right)+4\left(\frac{1}{\sqrt{5+6}}\right)+4\left(\frac{1}{\sqrt{5+7}}\right)+$ $8\left(\frac{1}{\sqrt{5+8}}\right)+(4 n-12)\left(\frac{1}{\sqrt{6+6}}\right)+(16 n-48)\left(\frac{1}{\sqrt{6+8}}\right)+4\left(\frac{1}{\sqrt{7+8}}\right)+6\left(\frac{1}{\sqrt{7+10}}\right)+$ $(8 n-14)\left(\frac{1}{\sqrt{8+8}}\right)+(32 n-100)\left(\frac{1}{\sqrt{8+10}}\right)+\left(15 n^{2}-96 n+152\right)\left(\frac{1}{\sqrt{10+10}}\right)$. $=\frac{1}{10}\left(15 n^{2}-96 n+152\right) \sqrt{5}+\frac{1}{6}(32 n-100) \sqrt{2}+\frac{1}{14}(16 n-48) \sqrt{14}+\frac{1}{6}(4 n-$ 12) $\sqrt{3}+2 n-\frac{7}{2}+\frac{6}{17} \sqrt{17}+\frac{4}{15} \sqrt{15}+\frac{8}{13} \sqrt{13}+\frac{4}{11} \sqrt{11}+\frac{4}{3} \sqrt{3}+\sqrt{10}+\frac{1}{2} \sqrt{2}$.

(8) $G A(G)=\sum_{u v \in E(G)} \frac{2 \sqrt{d_{u} d_{v}}}{d_{u}+d_{v}}$

$=2 \frac{2 \sqrt{4 \times 4}}{4+4}+8 \frac{2 \sqrt{4 \times 6}}{4+6}+4 \frac{2 \sqrt{4 \times 8}}{4+8}+2 \frac{2 \sqrt{5 \times 5}}{5+5}+4 \frac{2 \sqrt{5 \times 6}}{5+6}+4 \frac{2 \sqrt{5 \times 7}}{5+7}+8 \frac{2 \sqrt{5 \times 8}}{5+8}+$ $(4 n-12) \frac{2 \sqrt{6 \times 6}}{6+6}+(16 n-48) \frac{2 \sqrt{6 \times 8}}{6+8}+4 \frac{2 \sqrt{7 \times 8}}{7+8}+6 \frac{2 \sqrt{7 \times 10}}{7+10}+(8 n-14) \frac{2 \sqrt{8 \times 8}}{8+8}+$ $(32 n-100) \frac{2 \sqrt{8 \times 10}}{8+10}+\left(15 n^{2}-96 n+152\right) \frac{2 \sqrt{10 \times 4}}{10+10}$.

$=15 n^{2}+\frac{4}{9}(32 n-100) \sqrt{5}+\frac{4}{7}(16 n-48) \sqrt{3}-84 n+\frac{12}{17} \sqrt{70}+\frac{16}{15} \sqrt{14}+$ $\frac{32}{13} \sqrt{10}+\frac{2}{3} \sqrt{35}+\frac{8}{11} \sqrt{30}+\frac{8}{3} \sqrt{2}+\frac{16}{5} \sqrt{6}+130$.

(9) $A Z I(G)=\sum_{u v \in E(G)}\left(\frac{d_{u} d_{v}}{d_{u}+d_{v}-2}\right)^{3}$

$=2\left(\frac{4 \times 4}{4+4-2}\right)^{3}+8\left(\frac{4 \times 6}{4+6-2}\right)^{3}+4\left(\frac{4 \times 8}{4+8-2}\right)^{3}+2\left(\frac{5 \times 5}{5+5-2}\right)^{3}+4\left(\frac{5 \times 6}{5+6-2}\right)^{3}+4\left(\frac{5 \times 7}{5+7-2}\right)^{3}+$ $8\left(\frac{5 \times 8}{5+8-2}\right)^{3}+(4 n-12)\left(\frac{6 \times 6}{6+6-2}\right)^{3}+(16 n-48)\left(\frac{6 \times 8}{6+8-2}\right)^{3}+4\left(\frac{7 \times 8}{7+8-2}\right)^{3}+$ $6\left(\frac{7 \times 10}{7+10-2}\right)^{3}+(8 n-14)\left(\frac{8 \times 8}{8+8-2}\right)^{3}+(32 n-100)\left(\frac{8 \times 10}{8+10-2}\right)^{3}+\left(15 n^{2}-96 n+\right.$ $152)\left(\frac{10 \times 10}{10+10-2}\right)^{3}$

$=\frac{625000}{243} n^{2}-\frac{109249838528}{10418625} n+\frac{1427097955303080667}{133703165756160}$.

TABle 2. The size partition of $G$

\begin{tabular}{|c|c|c|c|c|c|}
\hline$\left(d_{u}, d_{v}\right)$ where $u v \in E(G)$ & $(4,4)$ & $(4,6)$ & $(4,8)$ & $(5,5)$ & $(5,6)$ \\
\hline Number of edges & 2 & 8 & 4 & 2 & 4 \\
\hline$\left(d_{u}, d_{v}\right)$ where $u v \in E(G)$ & $(5,7)$ & $(5,8)$ & $(6,6)$ & $(6,8)$ & $(7,8)$ \\
\hline Number of edges & 4 & 8 & $4 n-12$ & $16 n-48$ & 4 \\
\hline$\left(d_{u}, d_{v}\right)$ where $u v \in E(G)$ & $(7,10)$ & $(8,8)$ & $(8,10)$ & $(10,10)$ & \\
\hline Number of edges & 6 & $8 n-14$ & $32 n-100$ & $15 n^{2}-96 n+152$ & \\
\hline
\end{tabular}

\section{Competing Interests}

The author(s) do not have any competing interests in the manuscript. 


\section{REFERENCES}

1. Randić, M. (1975). Characterization of molecular branching. Journal of the American Chemical Society, 97(23), 6609-6615.

2. Bollobs, B., \& Erds, P. (1998). Graphs of extremal weights.Ars Combinatoria, 50, 225-233.

3. Gutman, I., \& Trinajstic, N. (1972). Graph theory and molecular orbitals. Total $\varphi$-electron energy of alternant hydrocarbons. Chemical Physics Letters, 17(4), 535-538.

4. Todeschini, R., \& Consonni, V. (2008). Handbook of molecular descriptors (Vol. 11). John Wiley \& Sons.

5. Fath-Tabar, G. H. (2011). Old and new Zagreb indices of graphs. MATCH Commun. Math. Comput. Chem, 65, 79-84.

6. Shirdel, G. H., Rezapour, H., \& Sayadi, A. M. (2013). The hyper-Zagreb index of graph operations. Iranian Journal of Mathematical Chemistry, 4(2), 213-220.

7. Estrada, E., Torres, L. Rodriguez, L., \& Gutman, I. (1998) An atom-bond connectivity index, Modelling the enthalpy of formation of alkanes. Indian J. Chem. 37, 849-855.

8. Furtula, B., Graovac, A., \& Vukicevic, D. (2009). Atombond connectivity index of trees.Discrete Applied Mathematics,157(13), 2828-2835.

9. Zhou, B., \& Trinajstic, N. (2010). On general sum-connectivity index. Journal of mathematical chemistry, 47(1), 210-218.

10. Zhou, B., \& Trinajstic, N. (2009). On a novel connectivity index. Journal of mathematical chemistry, 46(4), 1252-1270.

11. Vukicevic, D., \& Furtula, B. (2009). Topological index based on the ratios of geometrical and arithmetical means of end-vertex degrees of edges. Journal of mathematical chemistry, 46(4), 1369-1376.

12. Furtula, B., Graovac, A. \& Vukievi, D. (2010). Augmented Zagreb index. J. Math. Chem. $48,370-380$

13. Perc, M., Gmez-Gardees, J., Szolnoki, A., Flora, L. M., \& Moreno, Y. (2013). Evolutionary dynamics of group interactions on structured populations: a review. Journal of the royal society interface, 10(80), 20120997.

14. Gutman, I., \& Das, K. C. (2004). The first Zagreb index 30 years after. MATCH Commun. Math. Comput. Chem, 50, 83-92. 


\section{Hafiz Mutee ur Rehman}

Division of Science and Technology, University of Education, Lahore Pakistan. e-mail: rehman.mutee@yahoo.com

\section{Riffat Sardar}

Department of Mathematics, University of Sargodha, Lahore Pakistan.

e-mail: riffatmath@gmail.com

\section{Ali Raza}

Department of Mathematics, The University of Lahore, Lahore Pakistan. e-mail: ali-pu2002@yahoo.com 